\title{
14q22q23 microdeletion syndrome
}

INSERM

\section{Source}

INSERM. (1999). Orphanet: an online rare disease and orphan drug data base. 14q22q23 microdeletion syndrome. ORPHA:264200

$14 q 22 q 23$ microdeletion syndrome is a rare partial deletion of the long arm of chromosome 14 characterized by ocular anomalies (anopthalmia/microphthalmia, ptosis, hypertelorism, exophthalmos), pituitary anomalies (pituitary hypoplasia/aplasia with growth hormone deficiency and growth retardation) and hand/foot anomalies (polydactyly, short digits, pes cavus). Other clinical features may include muscular hypotonia, psychomotor development delay/intellectual disability, dysmorphic signs (facial asymmetry, microretrognathia, high-arched palate, ear anomalies), congenital genitourinary malformations, hearing impairment. Smaller 14q22 deletions may have variable expression. 